\title{
Confirmation of Microbial Ingress from Space
}

\author{
N. C. Wickramasinghe ${ }^{1,2,3^{*}}$, M. J. Rycroft ${ }^{4.5}$, D.T. Wickramasinghe ${ }^{2,6}$, E, J. Steele ${ }^{2,7}$, Daryl. H. Wallis ${ }^{3}$, \\ Robert Temple $^{8}$, G. Tokoro ${ }^{2,3}$, A.V. Syroeshkin ${ }^{10}$, T.V. Grebennikova ${ }^{9,8}$, O.S. Tsygankov ${ }^{11}$ \\ ${ }^{1}$ Buckingham Centre for Astrobiology, University of Buckingham, Buckingham MK18 1EG, UK, \\ ${ }^{2}$ Centre for Astrobiology, University of Ruhuna, Matara, Sri Lanka, \\ ${ }^{3}$ Institute for the Study of Panspermia and Astrobiology, Gifu, Japan, \\ ${ }^{4}$ Department of Electronic and Electrical Engineering, University of Bath, Bath BA2 7AY, UK, \\ ${ }^{5}$ CAESAR Consultancy, 35 Millington Road, Cambridge CB3 9HW, UK, \\ ${ }^{6}$ College of Physical and Mathematical Sciences, Australian National University, Canberra, Australia, \\ ${ }^{7} \mathrm{CY}$ O'Connor ERADE Village Foundation, Piara Waters,WA, Australia, \\ ${ }^{8}$ The History of Chinese Culture Foundation, Conway Hall, London, UK, \\ ${ }^{9}$ Federal Research Center of Epidemiology and Microbiology named Gamalei, Moscow, Russian Federation, \\ ${ }^{10}$ RUDN University, 6 Miklukho-Maklaya St, Moscow, 117198, Russian Federation, \\ ${ }^{11}$ Korolev Rocket and Space Corporation «Energia», Korolev, Russian Federation. \\ Email: ncwick@gmail.com
}

\begin{abstract}
The discovery by Russian researchers (Grebennikova et al., 2018) of microorganisms on the exterior surface of the International Space Station (ISS) on several occasions between 2013 and 2017 may be interpreted as evidence supporting the Hoyle-Wickramasinghe theory of cometary panspermia. The homologies between the ISS-recovered genotypes and known terrestrial bacteria can be seen as evidence of co-evolution and gene transfers (HGT) within a biosphere that spans astronomical distances. On the one hand, the height of the ISS orbit at $400 \mathrm{~km}$ can be argued as being too high for lofting surface microorganisms. However, there is a theory that purports to explain the possible transport of small particles from the troposphere into the lower stratosphere and ionosphere as a result of vortex motions as well as vertical flows (streamers), which are generated as a result of the development of the modulational instability in the ionospheric plasma. More research is needed to properly evaluate this proposal.
\end{abstract}

Keywords: Panspermia, cosmic biology, bacteria, Earth-environment, International Space Station

\section{Introduction}

The theory of cometary panspermia predicts that bacteria and viruses (and even other living systems in protective matrices) arrive at the Earth continuously as well as, more dramatically and far less frequently, in sporadic bursts (Arrhenius, 1903, 1908; Hoyle and Wickramasinghe, 1981, 1982, 1985, 2000). The latter may have happened with comet impacts that took place during the Cambrian explosion of multicellular life some 540Mya, and also at the time of the $\mathrm{K} / \mathrm{T}$ extinction and diversification event at 65Mya. The implication of cometary panspermia is that the terrestrial biosphere at a microbial level is not confined to the Earth but extends over vast astronomical dimensions. The origin and evolution of life on the Earth must accordingly involve genetic exchanges and interactions that extend well beyond the confines of our planet. Furthermore, the well-attested process of Horizontal Gene Transfer (HGT) that is normally regarded as being confined to the Earth must now be extended to embrace a cosmic domain (Wickramasinghe, 2013). In this paper we review the implications of recent studies that appear to give evidence of an ongoing entry of biological cells to the Earth from space.

\section{Searching for Cometary Microbes in the Stratosphere and High Atmosphere}

Serious attempts to test the possibility of biological entities (bacteria and viruses) entering the Earth's 
atmosphere from above have been made from the dawn of the present millennium. In 2001, samples of the stratosphere recovered from a height of $41 \mathrm{~km}$ were found to contain both culturable as well as viable but non-culturable bacteria (Harris et al., 2002; Narlikar et al., 2002; Wainwright et al., 2005). The maximum height of these collections, $41 \mathrm{~km}$, was significantly higher than the tropopause which is a ceiling for micron-sized atmospheric dust, so a prima facie case for the possible non-terrestrial origin was made. However, mechanisms to transport micron-sized dust from the ground to $41 \mathrm{~km}$ could not be fully excluded, so this discovery (as well as later discoveries of bacteria in the stratosphere) have all remained inconclusive with respect to their possible space origin (Shivaji et al., 2009; Wainwright et al., 2015).

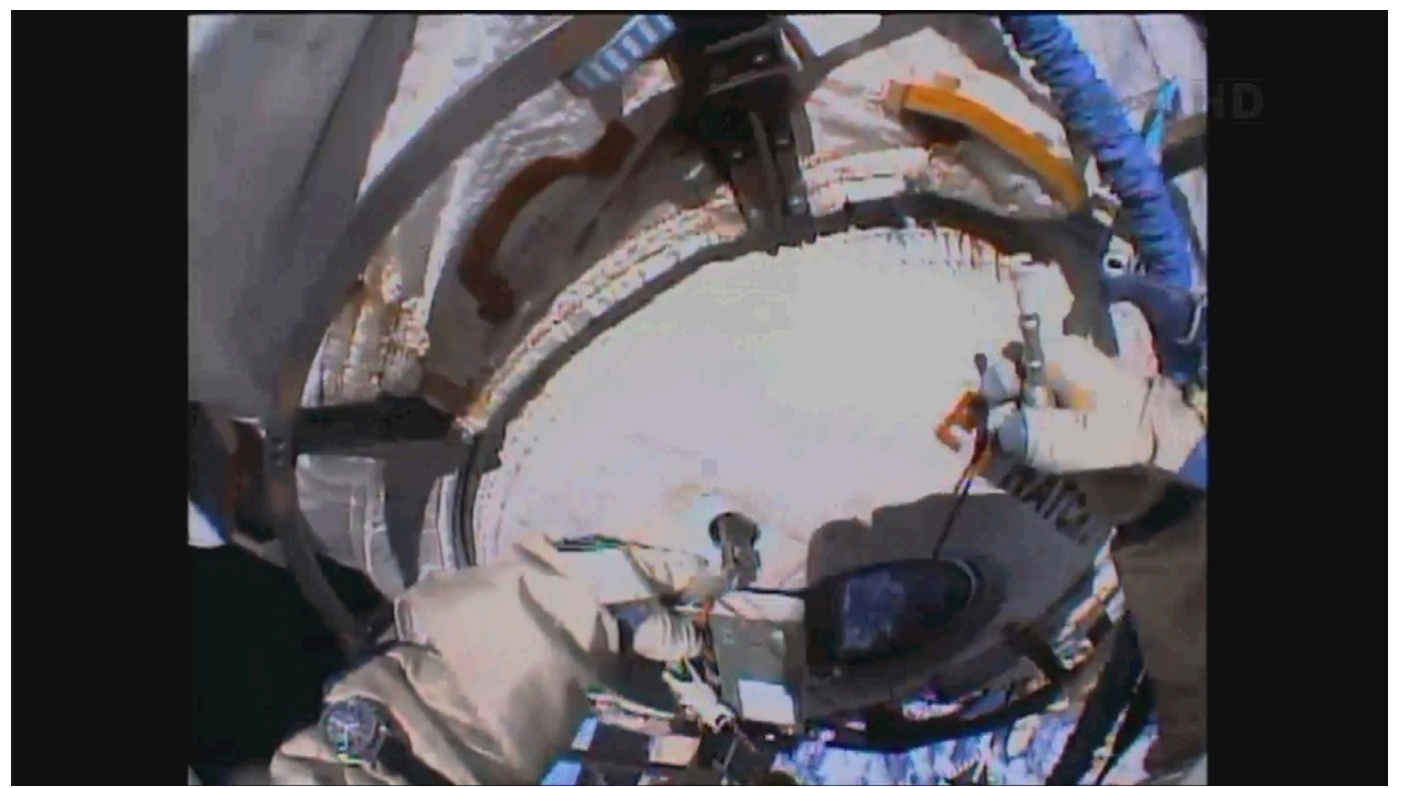

Figure 1. Illuminator of the ISS from which samples of cosmic dust were aseptically recovered

The most recent investigations by Russian researchers (Grebennikova et al., 2018) are highly relevant in the present context. A team of cosmonauts working at the International Space Station (ISS) obtained samples of cosmic dust from the exterior (illuminator) of the spacecraft in a series of separate collections between 2013 and 2017 (see Fig. 1). The samples were collected under the most stringent conditions to avoid contamination, and subsequent laboratory studies on Earth were also conducted aseptically with the highest degree of biological protection.

In six separate collections spanning a 6-year period, and experiments involving the polymerase chain reaction (PCR), DNA sequencing and phylogenetic analysis, Grebennikova et al. (2018) established the presence of bacteria of the genus Mycobacteria and the extremophile genus Delftia, amongst others, associated with deposits of cosmic dust. The similarity of the discovered genotypes with terrestrial bacteria could suggest a possible terrestrial origin, but elevation of terrestrial bacteria and dust to the height of the International Space Station at $400 \mathrm{~km}$ presents serious problems. Wickramasinghe and Rycroft (2018) have argued that electrically charged dust/bacteria cannot be transported to these heights by any known physical process. Likewise, the fact that DNA recoveries were made on several occasions well separated between spacewalks and sterilization events militates against the possibility of artificial contamination. If contamination is definitely ruled out, the results of Grebennikova et al. (2018) may provide the most powerful support thus far for ongoing panspermia.

On the other hand, there are articles which claim to show that the transfer of small particles from the troposphere into the lower stratosphere can be accomplished by slowly rotating synoptic vortices that are constantly present in the atmosphere and can reach stratospheric heights (Besedinaa et al., 2009). It has been argued that one of the means of transport of dust particles in the ionosphere is via vertical flows (streamers) which are generated as a result of the development of the modulational instability in the ionospheric plasma (Benkadda et al., 2011). Layers of dust particles in the ionosphere about a kilometer thick, formed at altitudes less than $120 \mathrm{~km}$, are posited to spread in the field of vortical 
structures. As a result, dust vortices may appear at an altitude of 110-120 km, and it becomes possible to transport particles to altitudes of $130 \mathrm{~km}$ and perhaps even higher (Popel et al., 2010, 2011; Benkadda et al., 2011). Because the implications of the discovery of an extraterrestrial input of microbiota for evolutionary biology as well as for epidemiology are so profound and far-reaching, it is most desirable that this mechanism of transport must be demonstrated - both theoretically and experimentally - to be effective or not, as the case may be. If it is the case that there is a viable mechanism that can uplift biological dust up to $400 \mathrm{~km}$ some fraction of this material will also escape Earth's gravity. In this case one would have an interesting demonstration of reverse panspermia, the life-laden Earth itself polluting the Solar system outside and the galaxy beyond. A strong argument against, however, is the fact the DNA sequences isolated from the ISS swabs do not refer to any common terrestrial microbiota such as are commonly found on the Earth's surface or in the troposphere.

\section{Cosmic Dust in the Stratosphere}

The flux of cosmic dust reaching the Earth from the zodiacal dust cloud and directly from comets has been estimated in numerous recent studies to exceed $\sim 100$ tonnes per day over the entire Earth (Plane et al., 2016). Much of this material is expected to cross the orbit of the ISS (at a height of $400 \mathrm{~km}$ ). However, Plane et al. (2016) estimate that only $\sim 6$ tonnes per day of such dust particles survive ablation lower in the mesosphere and reach the ground intact. If only one part in a billion of the surviving mass of material is biological in nature (bacteria) the total microbial biomass daily added to the Earth's environment is equivalent to $\sim 10^{12}$ bacterial cells.

In the work of Grebennikova et al. (2018) the illuminator (window) of the ISS at 400km would have intercepted the undiluted flux of cosmic dust. Assuming an effective window area that collects the incoming dust to be $\sim 0.2 \mathrm{~m}^{2}$, a global influx of 100 tonnes per day is equivalent to $\sim 4.6 \times 10^{-17} \mathrm{~g} \mathrm{~cm}^{-2} \mathrm{~s}^{-1}$ at the height of the ISS. Over a period of about a month a window presenting an average cross-section of $\sim 0.2 \mathrm{~m}^{2}$ is easily shown to capture $1.2 \times 10^{-6} \mathrm{~g}$ of cosmic dust per month (assuming unit sticking probability). Even if only $0.01 \%$ of this material is in the form of bacteria, we find that of the order of a $10^{5}$ bacterial cells will arrive on the $0.2 \mathrm{~m}^{2}$ window area in a month. Of course, additional studies are needed to reveal how viable the bacteria will be in the radiation conditions of space (both electromagnetic - X- and gamma-radiation - and corpuscular - galactic cosmic rays).

\section{New Paradigm}

Finally we note that new knowledge has been obtained for changing the reigning paradigm in science; this is the detection of the DNA of microorganisms on the external surface of the ISS. There are researchers who believe that there is no natural mechanism for raising microbes from the Earth up to a height of $400 \mathrm{~km}$, and not only for one isolated case, but six times in the period 2013-2017. There are other researchers who are considering complex mechanisms for raising dust particles from the Earth's surface to the ISS orbit.

The mechanism producing the upward movement of dust particles must be separately proven. But one thing is clear: we can no longer think from the point of view of the earthly life that arose on Earth and with its biosphere being bounded by our planet. Life is really an interconnected biosystem that includes all the stars and planets of the Milky Way. Evolution, including human evolution, should now be seen as a net result of the exchange of genes in the form of bacteria and viruses over cosmological distance scales.

Our new perspective in no way hinders or contradicts the enormous technical progress achieved in all areas of modern biology, including epidemiology. However, it is necessary to expand the sphere of evolutionary biology at all levels, including in it links with the "biosphere" of astronomical dimension. Whether this biosphere is limited to our galaxy, our local group of galaxies or beyond is a matter still to be discussed. The recent review of the extant cosmic life evidence from the biomedical perspective attests to this change in thinking (Steele et al., 2018).

\section{Conclusion}


In order to stress the vital importance of the new discoveries discussed in this paper we cannot do better than quote from Hoyle and Wickramasinghe (2003):

"The multiplying capacity of bacteria is enormous...... To go from an individual bacterium to the number of all the interstellar grains requires about 170 doublings. When conditions are optimal a bacterial population can double in a few hours, so 170 doublings would take less than a month. Of course such a prodigious explosion in number would never literally be achieved because of practical limitations occurring in the availability of nutrients. Nevertheless, there is evidence that whole galaxies are overwhelmed from time to time by comparatively rapid and very large scale episodes of grain formation.......

For a generation or more, astronomers have been accustomed to thinking of star-forming episodes accompanied by the production of vast clouds of interstellar grains. The episodes are sometimes local but they are often galaxy-wide. They are thought to be triggered by some large-scale event, the after effects of which linger on for some considerable time, several hundred million years. The condensation of the exceptionally bright stars which delineate the spiral structures of galaxies has often been associated with these episodes. From our argument it seems that even the origin of the spiral structures of galaxies may well be biological in nature.

The potential of bacteria to increase vastly in their number is enormous. It should occasion no surprise, therefore, that bacteria are widespread throughout astronomy. Rather would it be astonishing if biological evolution has been achieved on the Earth alone, without the explosive consequences of such a miracle ever being permitted to emerge into the Universe at large. How could the Universe ever be protected from such a devastating development? This indeed would be a double miracle, first of origin, and second of terrestrial containment.

Some biologists have probably found themselves in opposition to our argument for the proprietary reason that it seemed as if an attempt were being made to swallow up biology into astronomy. Their ranks may now be joined by those astronomers who see from these last developments that a more realistic threat is to swallow up astronomy into biology." It is into this intellectual knot that the new discipline of astrobiology was born.

\section{References}

1. Arrhenius, S., 1903. Die Verbreitung des Lebens im Weltenraum. Die Umschau, 7, pp. 481-485.

2. Arrhenius, S., 1908. Worlds in the Making. London: Harper.

3. Benkadda S., D.N. Klochkov, S.I. Popel, and Yu.N. Izvekova, 2011, Nonlinear excitation of zonal flows and streamers in plasmas. Physics of Plasmas 18, 052306, 11pp.

4. Besedinaa Yu.N., S.I. Popel, and P.K. Shuklab, 2009, Vortex Motions and Transportation of Fine Disperse Dust Particles in the Ionosphere (ISSN 1028334X), Doklady Earth Sciences, 429 (No. 8), pp. 1407-1410.

5. Grebennikova, T.V., Syroeshkin, A.V., Shubralova, E.V. et al, 2018. The DNA of bacteria of the World Ocean and the Earth in cosmic dust at the International Space Station, The Scientific World Journal, Volume 2018 (2018), Article ID 7360147, 7 pp. https://doi.org/10.1155/2018/7360147

6. Harris, M.J., et al., 2002. The Detection of Living Cells in the Stratosphere. Proceedings of the SPIE Conference, 4495, 192-198

7. Hoyle, F. and Wickramasinghe, N.C., 1981. In: C. Ponnamperuma, ed. Comets and the Origin of Life. Dordrecht: D. Reidel, pp. 227

8. Hoyle, F. and Wickramasinghe, N.C., 1982. Proofs that Life is Cosmic. Colombo: Govt. Press, Sri Lanka

9. Hoyle, F. and Wickramasinghe, N.C., 1985. Living Comets. Cardiff: Univ. College, Cardiff Press

10. Hoyle, F. and Wickramasinghe, N.C., 2000. Astronomical Origins of Life: Steps towards Panspermia. Kluwer Academic Press

11. Hoyle, F and Wickramasinghe, N.C., 2003. Astronomy or Biology?, Astrophys.Sp.Sci., 285, 539-554

12. Plane, J., Diego, J., Gomez-Martin, J.C. et al, 2016. How large is the cosmic dust flux into the Earth's atmosphere? 41st COSPAR Scientific Assembly, Abstract C5.2-5-16. http://adsabs.harvard.edu/abs/ 2016cosp...41E1569P

13. Popel S I, SI Kopnin, M Y Yu, J X Ma and Feng Huang, 2011. The effect of microscopic charged particulates in space weather J. Phys. D: Appl. Phys. 44, 174036- 174043 
14. Popel S.I., Yu.N. Izvekova and P.K. Shukla, 2010. Nano- and Microscale Particles in Vortex // Motions in Earth's Atmosphere and Ionosphere. New Frontiers in Advanced Plasma Physics, 189-201

15. Shivaji, S., Chaturvedi, P., Begum, Z., et al, 2009. Janibacter hoylei sp. nov., Bacillus isronensis sp. nov. and Bacillus aryabhattai sp. nov., isolated from cryotubes used for collecting air from the upper atmosphere. Int $J$ Syst Evol Microbiol, 59, 2977-2986

16. Steele E.J., Al-Mufti, S., Augustyn, K.A., Chandrajith, R., Coghlan, J.P., Coulson, S.G., Ghosh, S., Gillman, M., Gorczynski, R.M., Klyce, B., Louis, G., Mahanama, K., Oliver, K.R., Padron, J., Qu, J., Schuster, J.A., Smith, W.E., Snyder, D.P., Steele, J.A., Stewart, B.J., Temple, R., Tokoro, G., Tout, C.A., Unzicker, A., Wainwright, M., Wallis, J., Wallis, D.H., Wallis, M.K., Wetherall, J., Wickramasinghe, D.T., Wickramasinghe, J.T., Wickramasinghe, N.C., and Liu, Y., 2018. Cause of Cambrian Explosion - Terrestrial or Cosmic, Prog Biophys Mol Biol, 136, 3-23

17. Wainwright, M., Wickramasinghe, N., Narlikar, J., and Rajaratnam, P., 2003. Microorganisms cultured from stratospheric air samples obtained at 41km, FEMS Microbiol Lett, 218, 161-165

18. Wainwright, M., Wickramasinghe, N.C., Narlikar, J.V. et al, 2004. Confirmation of the presence of viable but non-culturable bacteria in the stratosphere, Int. J. Astrobiology, 3(1), 13-15

19. Wainwright, M., et al, 2015. Biological entities isolated from two stratosphere launches - continued evidence for a space origin, J Astrobiol. Outreach, 3:2

20. Wickramasinghe, N.C., 2013. DNA sequencing and predictions of the cosmic theory of life, Astrophys Space Sci, 343(1), 1-5; https://arxiv.org/abs/1208.5035

21. Wickramasinghe, N.C. and Rycroft, M.J., 2018. On the Difficulty of the Transport of Electrically Charged Submicron Dust from the Earth's Surface to the High Ionosphere, Advances in Astrophysics 3 (3), 150-153; https://dx.doi.org/10.22606/adap.2018.33003 